\title{
Invariants d'une désingularisation et singularités des morphismes
}

\author{
Benoît Audoubert, Fouad El Zein et Lê Dũng Tráng
}

\begin{abstract}
Let $(X, 0)$ be a germ of an analytic space and $f$ the germ of an analytic function on $X$. We show that the polar filtration of the local Milnor fiber, defined by a projection on a complex disc, is diffeomorphic to a valuative filtration of the Milnor fiber called Hironaka's filtration, a result which links invariants associated with the singularities of the projection to those associated with a desingularisation.
\end{abstract}

\section{Introduction}

Soient $(X, 0)$ un germe d'espace analytique et $f$ un germe de fonction analytique sur $X$. La fibre de Milnor $F$ de $f$ en zéro [Mil68], généralisée à un germe singulier [Le77] est un invariant analytique de $f$. Une désingularisation $P: Z \longrightarrow X$ dans laquelle la fibre spéciale de $f$ est un diviseur $D$ à croisements normaux (DCN) et une projection stratifiée de $F$ sur $D$ permettent de reconstituer la topologie de la fibre de Milnor $F$. Cette technique, introduite par Grothendieck, a été développée par Clemens [Cle77, ch. II, § 6, p. 241]. Aussi, elle a été bien illustrée dans le livre de Brieskorn et Knörrer [BK86, ch. III, $\S 8.5$, p. 566]. Une autre technique introduite par Lê [Le77], [Le75] consiste à considérer une projection $g$ de $X$ sur un disque et à utiliser la courbe polaire relative de $f$ associée à $g$ et le discriminant polaire (ou diagramme de Cerf) pour en déduire une filtration polaire sur $F$.

Le but de cet article est d'obtenir une relation entre les invariants du discriminant polaire et ceux de la désingularisation généralisant les travaux sur les courbes de [LMW89, SZ85], Maugendre [Mau95] et Taher [Tah97]. Pour cela, notre résultat principal consiste à montrer que la filtration polaire sur la fibre de Milnor $F$ est difféomorphe à une filtration valuative que nous appelons filtration d'Hironaka obtenue à partir de valuations sur les composantes irréductibles du diviseur $D$. La technique centrale de cet article est une généralisation en dimension supérieure du plombage en dimension deux complexe (voir § 3). Cette généralisation utilise les résultats de Clemens sur F [Cle69]. Steenbrink et Zucker ont appelé filtration polaire ce que nous appelons ici filtration d'Hironaka. Ils démontrent, dans le cas où $X$ est $\mathbb{C}^{2}$, le difféomorphisme de leur filtration avec la filtration polaire définie par Lê. Nous le faisons en général. A l'aide de la théorie de Lefschetz qui remplace, dans les arguments, le théorème de Riemann-Hurwitz sur les revêtements, nous généralisons alors des résultats connus sur les courbes.

\section{Enoncé du théorème principal}

\subsection{Hypothèses}

Soit $\varphi=(f, g):(X, 0) \longrightarrow\left(\mathbb{C}^{2}, 0\right)$ un germe de morphisme analytique. On suppose le lieu singulier de $X$, noté $X_{s}$, inclus dans $f^{-1}(0)$. Ceci est équivalent à demander que la fibre de Milnor locale de $f$

Received 13 March 2002, accepted in final form 31 July 2002.

2000 Mathematics Subject Classification 14B05, 32S05, 32S55.

Keywords: singularities, local Milnor fiber, polar construction, resolution of singularities.

This journal is (C) Foundation Compositio Mathematica 2004. 


\section{B. Audoubert, F. El Zein et Lê Dũng Tráng}

est non singulière. On suppose que le lieu singulier de $g^{-1}(0)$ est inclus dans son intersection avec $f^{-1}(0)$. Ceci nous permettra de trouver une résolution adaptée à $f$ et $g$ qui induit un isomorphisme en dehors de la fibre spéciale $f^{-1}(0)$ afin de préserver la fibre de Milnor.

Le lieu critique de $\varphi$ sur $X \backslash X_{s}$ est défini à l'aide de la matrice Jacobienne par rapport à un système de coordonnées locales $x_{i}$ pour $i=1$ à $n$ par la condition sur le rang

$$
r g\left(\begin{array}{lll}
\partial f / \partial x_{1} & , \ldots, & \partial f / \partial x_{n} \\
\partial g / \partial x_{1} & , \ldots, & \partial g / \partial x_{n}
\end{array}\right) \leqslant 1 .
$$

On note $C(\varphi)$ le germe d'espace, adhérence dans $X$ du lieu critique de $\varphi$. On suppose que l'adhérence dans $\left(\mathbb{C}^{2}, 0\right)$ de l'image par $\varphi$ du lieu critique de $\varphi$ est un germe de courbe, noté $D(\varphi)$.

DÉfinition. Le discriminant polaire $\Delta(\varphi)$ de $\varphi$ est le germe de courbe défini par la réunion des composantes de $D(\varphi)$ différentes des deux axes $u=0$ et $v=0$.

Dans toute la suite, nous supposons l'hypothèse suivante satisfaite :

HypothÈse. Le morphisme $\varphi$ est descriptible au sens qu'un représentant bien choisi de $\varphi$ soit une fibration topologique localement triviale au-dessus de l'intersection de $\mathbb{C}^{2} \backslash(\Delta(\varphi) \cup(u v=0)$ ) avec un voisinage de 0 dans $\mathbb{C}^{2}$ assez petit.

Cette notion mise en relief par les travaux de Thom (voir [LT83] où le terme descriptible est utilisé) n'est pas satisfaite lorsque par exemple $\varphi$ est un morphisme d'éclatement. En se donnant un plongement local de $X$ dans $\mathbb{C}^{n}$, la restriction $g$ d'une projection générique de $\mathbb{C}^{n}$ dans $\mathbb{C}$ définit avec $f$ un morphisme descriptible qui vérifie les hypothèses précédentes [Le77].

\subsection{Les nombres polaires}

Le discriminant polaire $\Delta(\varphi)$ se décompose en une réunion de courbes irréductibles $\Delta_{\lambda}, \lambda \in \Lambda$. Chaque composante $\Delta_{\lambda}$ admet, dans les coordonnés $(u, v)$ de $\mathbb{C}^{2}$, un développement de Puiseux

$$
v=a_{\lambda} u^{r_{\lambda}}+\cdots ; \quad r_{\lambda} \in \mathbb{Q},
$$

qui commence par l'exposant $r_{\lambda}>0$. Ces nombres sont les nombres polaires de B. Teissier [Tei75] dans le cas où $X$ est non singulier et $g$ est une projection générale. Quand $X$ est un espace singulier quelconque et $g$ est une projection générale, ces exposants permettent de construire la filtration polaire [Le75, Le78].

Remarque. Nous retiendrons les nombres $r_{\lambda}$ distincts sous la forme d'une suite croissante appelée suite de nombres polaires de $\varphi:\left\{r_{1}<\cdots<r_{k}\right\}$.

Dans le cas où $X$ est $\mathbb{C}^{2}$ et $g$ est une forme linéaire générique, on montre [LMW89] que la suite de nombres polaires coïncide avec la suite des quotients d'Hironaka aux points de rupture d'une désingularisation de la courbe $f^{-1}(0)$, ce qui donne dans ce cas la relation évoquée dans l'introduction entre les singularités de $\varphi=(f, g)$ et certains invariants de la désingularisation.

\subsection{Filtration polaire}

Considérons $\varphi:(X, 0) \longrightarrow\left(\mathbb{C}^{2}, 0\right)$ satisfaisant les hypothèses faites ci-dessus. Soit $\Delta_{\lambda}, \lambda \in \Lambda$ les composantes du discriminant polaire $\Delta(\varphi)$ de $\varphi$, admettant des développements de Puiseux de la forme $v=a_{\lambda} u^{r_{\lambda}}+\cdots$ avec $r_{\lambda} \in \mathbb{Q}$. Choisissons deux nombres $A$ et $B$ tels que

$$
A<\inf _{\lambda \in \Lambda}\left(\left|a_{\lambda}\right|\right), \quad B>\sup _{\lambda \in \Lambda}\left(\left|a_{\lambda}\right|\right) .
$$

Comme $r_{\lambda}>0$, les nombres $a_{\lambda} u^{r_{\lambda}}$ convergent vers zéro lorsque $u$ tend vers zéro, avec une vitesse qui dépend de $r_{\lambda}$ ce qui, pour $|u|$ assez petit, permet d'ordonner dans $\mathbb{R}^{+}$les valeurs suivantes associées 


\section{INVARIANTS D'UNE DÉSINGULARISATION ET SINGULARITÉS DES MORPHISMES}

à la suite polaire

$$
A|u|^{r_{k}}<B|u|^{r_{k}}<A|u|^{r_{k-1}}<\cdots<A|u|^{r_{i}}<B|u|^{r_{i}}<\cdots<B|u|^{r_{1}} .
$$

Si $r_{i}<r<r_{i+1}$, on peut choisir $u$ assez petit pour avoir aussi les inégalités

$$
A|u|^{r_{i+1}}<B|u|^{r_{i+1}}<A|u|^{r}<B|u|^{r}<A|u|^{r_{i}}<B|u|^{r_{i}} .
$$

On peut choisir un représentant du germe $\varphi$ défini sur un voisinage $V$ de 0 dans $X$ sur un voisinage polydisque $\mathbf{D}^{\prime} \times \mathbf{D}$, où $\mathbf{D}$ est un disque $D(0, \eta)$ de rayon $\eta$ centré en 0 . On notera encore $\varphi$ ce représentant. La fibre de Milnor locale de $f$ est alors $F_{t}=\varphi^{-1}(t \times \mathbf{D})$. Pour $u=t$ constant, on introduit une filtration du disque $(t, v)$ où $|v|<\eta$ par des disques $D_{r}=D\left(0, B|t|^{r}\right)$.

Désignons les points de $\Delta(\varphi) \cap(u=t)$ par $v_{\lambda, j}(t)$, avec $\lambda \in \Lambda$ et $j \in J_{\lambda}$; ce sont les valeurs critiques de la restriction de $g$ à la fibre de Milnor $F_{t}$. D'après les hypothèses faites, la restriction de $g$ au-dessus du disque moins ces points est une fibration localement triviale.

DÉfinition. La filtration polaire $F_{t}(r)$ sur la fibre de Milnor est définie par

$$
F_{t}(r)=\left\{x \in F_{t}:|g(x)|<B|t|^{r}\right\} .
$$

\subsection{Filtration d'Hironaka}

Considérons une résolution $P: Z \longrightarrow X$ telle que l'image réciproque $D^{\prime}$ de la réunion $f^{-1}(0) \cup g^{-1}(0)$ soit un $D C N$ de composantes irréductibles $D_{i}, i \in I^{\prime}$. Ainsi $D=(f \circ P)^{-1}(0)$ est aussi un $D C N$ égal à la réunion de $D_{i}$ pour $i \in I \subset I^{\prime}$. On peut supposer d'après les hypothèses faites sur les singularités de $X$ et de $g^{-1}(0)$, que la restriction de $P$ à $Z \backslash D$ soit un isomorphisme $Z \backslash D \stackrel{\sim}{\longrightarrow} X \backslash f^{-1}(0)$. Généralisant la notion de voisinage tubulaire, le diviseur $D^{\prime}$ admet un voisinage régulier $U^{\prime}=\bigcup_{i \in I^{\prime}} U_{i}$ union de voisinages tubulaires ouverts $U_{i}$ de $D_{i}$ (voir [Cle77]). Il contient un voisinage régulier $U=\bigcup_{i \in I} U_{i}$ de $D$. Le morphisme $P$ étant propre, la fibre de Milnor $F_{t}=f^{-1}(t)$ pour $|t|$ assez petit coïncide à isomorphisme analytique complexe près avec $(f \circ P)^{-1}(t)$ et se trouve dans $U$. Nous construirons une projection stratifiée (ou morphisme d'effondrement) $\Pi: F_{t} \longrightarrow D$ de la fibre de Milnor sur le diviseur $D$ (cf. $\S 3$ ). Ce morphisme est largement commenté dans le cas des courbes dans le livre de Brieskorn et Knörrer [BK86] qui décrivent comment on peut retrouver la topologie de $F_{t}$ à partir du diviseur $D$, dans ce cas.

Nous allons voir le comportement de l'effondrement $\Pi$ par rapport à la filtration polaire de $F_{t}$ généralisant ainsi le cas d'un morphisme de $\left(\mathbb{C}^{2}, 0\right)$ dans $\left(\mathbb{C}^{2}, 0\right)$ déjà largement étudié.

Pour toute composante $D_{i}$ on introduit le quotient des valuations $v_{D_{i}}(f)$ et $v_{D_{i}}(g)$ des fonctions $f$ et $g$, égales à la multiplicité des zéros de ces fonctions le long de $D_{i}$. Soit $q(i)=v_{D_{i}}(g) / v_{D_{i}}(f)$. On appelle ce quotient le quotient d'Hironaka de $\varphi$ sur la composante $D_{i}$.

Définition. La filtration d'Hironaka de la fibre de Milnor $F_{t}$ de $f$ induite par la désingularisation $P: Z \longrightarrow X$ est définie, pour tout rationnel $r$, comme suit :

$$
F_{t}^{H}(r)=F_{t} \cap\left(\bigcup_{i \in I^{\prime}, q(i) \geqslant r} U_{i}\right) .
$$

Remarque. Pour $r \gg 0$ très grand, on remarque que l'on a l'inclusion $F_{t}^{H}(r) \subset \bigcup_{i \in I^{\prime} \backslash I} U_{i}$. En effet, pour $i \in I^{\prime} \backslash I$, une composante de $g^{-1}(0)$ qui n'est pas dans $f^{-1}(0)$ est indexée par $i \in I^{\prime} \backslash I$ avec un quotient $q(i)=\infty$; pour $r$ assez grand, le terme $F_{t}^{H}(r)$ de la filtration s'envoie par $g$ sur un voisinage de zéro et est difféomorphe à un voisinage tubulaire de $g^{-1}(0) \cap F_{t}$. Donc il est difféomorphe au terme correspondant de la filtration polaire. On verra que cette filtration peut être construite à difféomorphisme près à l'aide des quotients d'Hironaka. 


\section{B. Audoubert, F. El Zein et Lê Dũng Tráng}

\subsection{Résultat principal}

ThÉorème 1. Pour chaque $r \in \mathbb{Q}^{+}$, le terme $F_{t}(r)$ de la filtration polaire est homéomorphe par un difféomorphisme par morceaux au terme $F_{t}^{H}(r)$ de la filtration d'Hironaka.

Ce théorème sera conséquence de la proposition suivante :

Proposition 1. i) Pour $r \in \mathbb{Q}^{+}$, soient les diviseurs

$$
D(r)=\bigcup_{i \in I, q(i) \geqslant r} D_{i}, \quad D^{\star}(r)=D(r) \backslash \bigcup_{i \in I, q(i)<r} D_{i}
$$

et $F_{t}^{\infty}(r)=F_{t} \cap\left(\bigcup_{i \in I^{\prime} \backslash I, q(j)<r}\left(U_{i} \cap U_{j}\right)\right)$. Il existe une projection stratifiée $\Pi: F_{t} \longrightarrow D$ de la fibre de Milnor $F_{t}$ de $f$, pour $|t|$ assez petit, sur le diviseur $D=(f \circ P)^{-1}(0)$ dans la désingularisée $Z$ de $X$ telle que $F_{t}(r)$ et $F_{t}^{H}(r)$ soient homéomorphes par un difféomorphisme par morceaux à $\Pi^{-1}\left(D^{\star}(r)\right) \cup F_{t}^{\infty}(r)$.

ii) Pour $r \in \mathbb{Q}^{+}$, considérons la couronne polaire

$$
C(r)=\left\{z \in \mathbf{D}: A|t|^{r}<|z|<B|t|^{r}\right\} .
$$

Il existe une isotopie stratifiée entre

$$
g^{-1}(C(r)) \cap F_{t} \quad \text { et } \quad F_{t} \cap\left(\bigcup_{q(i)=r} U_{i} \cup\left(\bigcup_{q(k)>r, q(j)<r} U_{k} \cap U_{j}\right)\right) .
$$

En particulier on en déduit un homéomorphisme et même un difféomorphisme par morceaux.

De plus l'image réciproque par la projection $g \mid F_{t}: F_{t} \longrightarrow \mathbf{D}$ de la couronne polaire se rétracte par déformation sur $\Pi^{-1}(\underline{D(r))})$ où

$$
\underline{D(r)}=\left(\bigcup_{q(i)=r} D_{i}\right) \cup\left(\bigcup_{q(k)>r, q(j)<r} D_{k} \cap D_{j}\right) .
$$

Avant de démontrer la proposition et le théorème, il est important d'énoncer les résultats de Clemens [Cle69] dans un langage adapté à nos besoins.

\section{Plombage et rétraction sur la fibre centrale}

\subsection{Voisinage régulier d'un DCN}

Soit $Y$ un DCN dans une variété analytique complexe $X$ de dimension complexe $n$ et soit $Y=\bigcup_{i \in I} Y_{i}$ la décomposition en diviseurs irréductibles $Y_{i}$ que nous supposons lisses. Le résultat suivant peut être extrait des travaux de Clemens [Cle77], [Cle69, § 3.4].

Théorème 2. Pour toute partie $J$ de l'ensemble d'indices $I$, soit $Y_{J}=\cap_{i \in J} Y_{i}$ et soit $N_{Y_{J} \mid X}$ le fibré normal de $Y_{J}$ dans $X$. Alors il existe un voisinage tubulaire $U_{J}$ de $Y_{J}$ dans $X$ et une projection $C^{\infty}$,

$$
\Pi_{J}: U_{J} \longrightarrow Y_{J}
$$

telle que :

i) Les fibres de $\Pi_{J}$ sont des sous-variétés holomorphes de $X$;

ii) Il existe des plongements ouverts $C^{\infty}, f_{J}: U_{J} \hookrightarrow N_{Y_{J} \mid X}$ au-dessus de $Y_{J}$ dont les restrictions sont holomorphes sur les fibres et tels que $Y_{J}$ s'envoie sur la section zéro ;

iii) Pour tout $J, K$ dans $I$, nous avons $U_{J} \cap U_{K}=U_{J \cup K}$ et si $K \subset J$ alors $\Pi_{J} \circ \Pi_{K}=\Pi_{J}$ sur $U_{J}$; 
iv) Soit $J_{i}=J \backslash\{i\}$ pour $i \in J$, alors on a un isomorphisme de $N_{Y_{J} \mid X}$ avec le produit fibré $\times_{i \in J}\left(N_{Y_{J} \mid Y_{J_{i}}} \longrightarrow Y_{J}\right)$ qui induit via $f_{J}$ un isomorphisme de $\left(U_{J} \longrightarrow Y_{J}\right)$ avec le produit fibré $\times_{i \in J}\left(U_{J} \cap Y_{J_{i}} \longrightarrow Y_{J}\right)$;

v) Les fibrés normaux $N_{Y_{J} \mid X}$ peuvent être munis de métriques hermitiennes de sorte que les ouverts $U_{J}$ soient des fibrés en polydisques de multirayons constants et que les matrices de changement de trivialisations soient diagonales complexes unitaires différentiables ;

vi) Si $f: U \rightarrow D$ est une application holomorphe dans un disque de fibre $Y$ en 0 et si on considère des coordonnées au voisinage d'un point a de $Y_{J}$ telles que $f$ s'écrive $f=z_{1}^{n_{1}} \cdots z_{p}^{n_{p}}$, alors on peut trouver des coordonnées $\left(y, v_{1}, \ldots, v_{p}\right)$ de $N_{Y_{J} \mid X}$, où les $v_{i}$ sont linéaires dans les fibres, telles que l'expression de $f$ soit $f=c(y) v_{1}^{n_{1}} \cdots v_{p}^{n_{p}}$, où $c(y)$ est une fonction à valeurs complexes différentiable en le système de coordonnées y de $Y_{J}$.

Remarque. On peut interpréter le résultat précédent comme une technique de plombage (différentiable) en dimension supérieure, connue en dimension complexe deux (voir [Mum61, p. 8]), qui consiste en particulier dans les données suivantes :

i) Un ensemble d'indices $I$ et une famille de variétés analytiques $Y_{K}$ indexées par les parties non vides $K$ de $I$;

ii) Une famille d'immersions fermées pour toute suite $J \subset K \subset I, \sigma_{J, K}: Y_{K} \hookrightarrow Y_{J}$ telles que $\forall L \subset J \subset K, L \neq \emptyset, \sigma_{L, J} \circ \sigma_{J, K}=\sigma_{L, K}$

iii) Des familles de fibrés complexes différentiables $N_{i}$ sur $Y_{i}, i \in I$, d'ouverts $U_{i} \subset N_{i}$ contenant la section nulle.

Ce qui permet de définir pour tout $J \subset I$ les ouverts $U_{J}$, destinés à devenir les voisinages réguliers de $Y_{J}$, comme l'espace total du produit fibré $\times_{i \in J}\left(\left(U_{i} \mid Y_{J}\right) \longrightarrow Y_{J}\right)$ des restrictions $U_{i} \mid Y_{J}$ de $U_{i}$ à $Y_{J}$.

Les données de recollement se traduiraient alors par :

iv) La donnée pour tout $j \in J$ de plongements ouverts de $U_{J_{j}} \mid Y_{J}$, la restriction de $U_{J_{j}}$ à $Y_{J}$, dans $Y_{j}$, prolongeant l'immersion de $Y_{J}$ dans $Y_{j}$. Soit $V_{J, j}$ l'image ;

v) La donnée d'isomorphismes entre la restriction de $U_{j}$ à $V_{J, j} \subset Y_{j}$ et $U_{J}$

$$
\left(U_{j} \mid V_{J, j}\right) \simeq\left(U_{j} \mid Y_{J}\right) \times_{Y_{J}}\left(U_{J_{j}} \mid Y_{J}\right) \simeq U_{J}=\times_{i \in J}\left(\left(U_{i} \mid Y_{J}\right) \longrightarrow Y_{J}\right)
$$

satisfaisant des conditions de compatibilité pour $K \subset J$.

Ces données devraient permettre de reconstituer un voisinage régulier de l'espace $Y=\cup_{i \in I} Y_{i}$ dans un espace ambiant $X$ obtenu par recollement des voisinages $U_{i}$ des $Y_{i}$. Bien que l'on ne puisse pas récupérer la structure analytique de $X$, ce résultat est utile dans la mesure où il explique pourquoi les démonstrations faites dans le cadre des fibrés normaux se recollent pour donner des résultats sur le voisinage $U$ de $Y$.

\subsection{Rétraction du voisinage régulier sur un DCN}

On va construire une projection $R: U \longrightarrow Y$ sur la réunion $Y$ des hyperplans coordonnées $Y_{i}: v_{i}=0$ d'un voisinage régulier de $Y$. Pour cela nous procédons par étapes. On fait d'abord une construction dans le cas réel de $\mathbb{R}^{n}$, puis dans le cas complexe de $\mathbb{C}^{n}$ et on utilisera le plombage pour recoller. Cette construction rend précise une idée de Thom (cf. [Tho70]).

3.2.1 Cas réel. Soit $\left(t_{1}^{\prime}, \ldots, t_{n}^{\prime}\right)$ les coordonnées canoniques (donc $\left.\geqslant 0\right)$ du quadrant $\left(\mathbb{R}^{+}\right)^{n}$. On note $Y^{\prime}$ la réunion des hyperplans de coordonnées $Y_{i}^{\prime}$ d'équation $t_{i}^{\prime}=0$. Pour tout sous-ensemble d'entiers $L \subset[1, n]$, on pose $Y_{L}^{\prime}=\bigcap_{j \in L} Y_{j}^{\prime}$ autrement dit

$$
Y_{L}^{\prime}=\left\{\left(t_{1}^{\prime}, \ldots, t_{n}^{\prime}\right): t_{i}^{\prime}=0, i \in L\right\}
$$




\section{B. Audoubert, F. El Zein et Lê Dũng Tráng}

et $Y_{L}^{\star \star}=Y_{L}^{\prime} \backslash \bigcup_{|M| \geqslant|L|} Y_{M}^{\prime}$. Remarquons que le quadrant $\left(\mathbb{R}^{+}\right)^{n}$ est naturellement stratifié par les $Y_{L}^{\prime \star}$ pour tous les sous-ensembles d'entiers de $[0, n]$ :

$$
\left(\mathbb{R}^{+}\right)^{n}=\bigcup_{L} Y_{L}^{\prime *}
$$

i) Strate de codimension $n, Y_{[1, n]}^{\prime}=\{0\}$. Soit $\left(K_{1}, \ldots, K_{n}\right)$ un point de $\left(\mathbb{R}^{+}\right)^{n}$. On considère le pavé $P^{\prime}\left(K_{*}\right)$ de $\left(\mathbb{R}^{+}\right)^{n}$ dont un sommet est l'origine et qui s'appuie sur les hyperplans de coordonnées et de côté de longueur $K_{i}$ sur l'axe des $t_{i}^{\prime}$. On note $\overline{P^{\prime}\left(K_{*}\right)}$ son adhérence dans $\left(\mathbb{R}^{+}\right)^{n}$. On note

$$
R^{\prime}: \overline{P^{\prime}\left(K_{*}\right)} \backslash\left(K_{1}, \ldots, K_{n}\right) \longrightarrow Y^{\prime}
$$

la projection conique de sommet $\left(K_{1}, \ldots, K_{n}\right)$.

Pour tout sous-ensemble d'entiers $L \subset[1, n]$, on distingue dans

$$
\overline{P^{\prime}\left(K_{*}\right)} \backslash\left(K_{1}, \ldots, K_{n}\right)
$$

les régions

$$
\begin{aligned}
Q_{L}^{\prime} & =R^{\prime-1}\left(Y_{L}^{\prime}\right) \\
& =\left\{\left(t_{1}^{\prime}, \ldots, t_{n}^{\prime}\right) \mid \frac{t_{i}^{\prime}}{K_{i}}=\frac{t_{j}^{\prime}}{K_{j}} \text { si } i, j \in L ; \frac{t_{j}^{\prime}}{K_{j}}>\frac{t_{i}^{\prime}}{K_{i}} \text { si } i \in L, j \notin L\right\} .
\end{aligned}
$$

On a

$$
\overline{P^{\prime}\left(K_{*}\right)}=\bigcup_{L \subset[1, n], L \neq \emptyset} Q_{L}^{\prime}
$$

Si on écrit $R^{\prime}=\left(R_{1}^{\prime}, \ldots, R_{n}^{\prime}\right)$, on a pour $u=\left(t_{1}^{\prime}, \ldots, t_{n}^{\prime}\right) \in Q_{L}^{\prime}, R_{j}^{\prime}(u)=0$ si $j \in L$ et $\left(R_{j}^{\prime}(u)\right)_{j \notin L}$ sont les coordonnées de $R^{\prime}(u)$ sur $Y_{L}^{\prime}$. La remarque importante pour la suite est que la restriction de cette projection à une face cö̈ncide avec la définition de la projection en dimension inférieure ce qui permet de recoller sur tout $\left(\mathbb{R}^{+}\right)^{n}$ ces projections avec celles que nous allons définir dans l'étape suivante.

ii) Strate de codimension $s$. Etant donné $J \subset[1, n]$, de longueur $|J|=s$, on considère le collier $U_{J}^{\prime}$ de $Y_{J}^{\prime}$ formé du produit de $Y_{J}^{\prime}$ par un pavé $P_{J}^{\prime}\left(K_{*}\right)$ de côtés de longueur $K_{i}$ dans l'axe de la coordonnées $t_{i}^{\prime}$, pour $i \in J$. On note $\overline{U_{J}^{\prime}}$ son adhérence dans $\left(\mathbb{R}^{+}\right)^{n}$. On considère $\overline{U_{J}^{\prime}}$ comme un pavé fibré sur $Y_{J}^{\prime}$. On note $Y_{J}^{\prime}\left(K_{*}\right)$ l'arête du pavé fibré $\overline{U_{J}^{\prime}}$ définie par $t_{j}^{\prime}=K_{j}$, pour $j \in J$. On a une projection conique à partir de cette arête

$$
R_{J}^{\prime}: \bar{U}_{J}^{\prime} \backslash Y_{J}^{\prime}\left(K_{*}\right) \longrightarrow \bigcup_{j \in J} Y_{j}^{\prime},
$$

définie fibre par fibre au-dessus de $Y_{J}^{\prime}$ en appliquant le cas précédent avec $n=s$.

iii) Recollement. La réunion $U^{\prime}=\bigcup_{i \in[1, n]} U^{\prime}{ }_{i}$ forme un collier de $Y^{\prime}$ sur lequel on construit la projection $R^{\prime}$ comme suit. On prend pour tout $J$ la restriction de $R_{J}^{\prime}$ à $\overline{U_{J}^{\prime}} \backslash\left(Y_{J}^{\prime}\left(K_{*}\right) \cup \hat{U}_{J}^{\prime}\right)$ où

$$
\hat{U}_{J}^{\prime}=\bigcup_{L \supset J} U_{L}^{\prime}=U_{J} \cap\left(\bigcup_{i \notin J} U_{i}\right) .
$$

Par construction même, les différentes restrictions des projections $R_{J}^{\prime}$ se recollent bien car, pour $J \subset L, R_{L}^{\prime}$ et $R_{J}^{\prime}$ coïncident sur le bord $\partial\left(\overline{U_{L}^{\prime}} \backslash\left(Y_{L}^{\prime}\left(K_{*}\right) \cup \hat{U}_{L}^{\prime}\right)\right)$. La projection $R^{\prime}$ est en fait la restriction d'une rétraction continue différentiable par morceaux. 
3.2.2 Cas complexe. On note $Y_{j}$ l'hyperplan tel que $z_{j}=0$ et $Y_{J}=\bigcap_{j \in J} Y_{J}$, où $J$ est un sous-ensemble d'entiers de $[1, n]$ :

$$
Y_{J}=\left\{\left(z_{1}, \ldots, z_{n}\right): z_{i}=0, i \in J\right\} .
$$

On note $Y_{J}^{\star}=Y_{J} \backslash \bigcup_{J \subset L, J \neq L} Y_{L}$. On remarque que $\mathbb{C}^{n}$ est stratifié par les $Y_{J}^{\star}$, pour tous les sous-ensembles d'entiers $J$ de $[1, n]$ :

$$
\mathbb{C}^{n}=\bigcup_{J \subset[1, n]} Y_{J}^{\star}
$$

On introduit l'application norme qui va servir à se ramener au cas réel

$$
N: \mathbb{C}^{n} \longrightarrow\left(\mathbb{R}^{+}\right)^{n}: N\left(z_{1}, \ldots, z_{n}\right)=\left(\left|z_{1}\right|, \ldots,\left|z_{n}\right|\right) .
$$

La restriction de $N$ au-dessus d'une strate ouverte $Y_{J}^{\prime \star}$ de codimension $|J|=s$ est une fibration en tores de dimension $s$. En effet, si l'on écrit $z_{j}=\rho_{j} e^{i \theta_{j}}$ où $\theta_{j} \in \mathbb{Z} / 2 \pi \mathbb{Z}$, une fibre de $N$ définie par une valeur fixée des $\rho_{j}$ est un produit de cercles.

On considère le voisinage régulier $U_{J}$ de $Y_{J}$, produit de $Y_{J}$ et d'un polydisque $P_{J}\left(K_{*}\right)$ de rayons $K_{i}$ dans l'axe de la $i$-ème coordonnée, pour $i \in J$ et d'adhérence $\overline{U_{J}}$.

Soit $Y_{J}\left(K_{*}\right)$ le tore distingué du polydisque défini par $\left|z_{j}\right|=K_{j}$, pour $j \in J$. On définit une projection complexe

$$
R_{J}: \overline{U_{J}} \backslash Y_{J}\left(K_{*}\right) \longrightarrow \bigcup_{j \in J} Y_{j}
$$

fibre par fibre au-dessus de $Y_{J}$, qui relève $R_{J}^{\prime}$, comme suit : pour

$$
\left(y, z_{j_{1}}, \ldots, z_{j_{s}}\right) \in Y_{J} \times P_{J}\left(K_{*}\right)=U_{J}
$$

avec

on pose

$$
z_{j .}=\left(z_{j_{1}}, \ldots, z_{j_{s}}\right)=\left(\left|z_{j_{1}}\right| e^{i \theta_{j_{1}}}, \ldots,\left|z_{j_{s}}\right| e^{i \theta_{j_{s}}}\right)
$$

$$
R_{J}\left(y, z_{j}\right)=\left(y, R_{j_{1}}^{\prime}\left(\left|z_{j_{1}}\right|, \ldots,\left|z_{j_{s}}\right|\right) e^{i \theta_{j_{1}}}, \ldots, R_{j_{s}}^{\prime}\left(\left|z_{j_{1}}\right|, \ldots,\left|z_{j_{s}}\right|\right) e^{i \theta_{j_{s}}}\right)
$$

où $R_{j_{k}}^{\prime}$ désigne la composante d'indice $j_{k} \in J$ de la projection réelle $R_{J}^{\prime}$ définie précédemment. Pour $L \supset J$ et $\left(\left|z_{j_{1}}\right|, \ldots,\left|z_{j_{k}}\right|, \ldots,\left|z_{j_{s}}\right|\right) \in Q_{L}^{\prime}$, la composante $R_{j}^{\prime}$ est égale à 0 quand $j \notin L$ et les autres composantes de $R_{J}$ sont les coordonnées sur $Y_{L}$. La réunion $U=\bigcup_{i \in[1, n]} U_{i}$ forme un voisinage régulier de $Y$ sur lequel on construit la projection $R$ comme suit. On prend pour tout $J$ la restriction de $R_{J}$ à $\overline{U_{J}} \backslash\left(Y_{J}\left(K_{*}\right) \cup \hat{U}_{J}\right)$ où

$$
\hat{U}_{J}=\bigcup_{L, J \subset L} U_{L}=U_{J} \cap\left(\bigcup_{i \notin J} U_{i}\right) .
$$

Les différentes restrictions des projections $R_{J}$ se recollent bien car pour $J \subset L, R_{L}$ et $R_{J}$ coïncident sur le bord $\partial\left(\overline{U_{L}} \backslash\left(Y_{J}\left(K_{*}\right) \cup \hat{U}_{L}\right)\right)$. La projection $R$ est en fait la restriction d'une rétraction continue différentiable par morceaux.

3.2.3 Cas général. Avec la technique de plombage, le cas général ne diffère pas du cas précédent. On considère un voisinage régulier $U=\cup_{i \in I} U_{i}$ de $Y$ formé d'ouverts dans les fibrés normaux $N_{Y_{i} \mid X}$ de rayon $K_{i}$ et d'adhérence $\overline{U_{i}}$. La norme $N: N_{Y_{J} \mid X} \longrightarrow Y_{J} \times\left(\mathbb{R}^{+}\right)^{s}$ où $s=|J|$ est une application au-dessus de $Y_{J}$ et les changements de cartes étant unitaires sont transparents pour la norme $N$. La définition de $R_{J}$ dans le cas complexe s'applique à une trivialisation du fibré. Elle est compatible avec les morphismes de recollement par les matrices diagonales unitaires. En effet dans l'isomorphisme $N_{Y_{J} \mid X} \simeq \times_{i \in J}\left(N_{Y_{i} \mid X} \mid Y_{J}\right)$ les hyperplans coordonnées du produit fibré $\times_{i \in J \backslash\{k\}}\left(N_{Y_{i} \mid X} \mid Y_{J}\right)$ s'identifient par l'isomorphisme du plombage aux hyperplans coordonnées $Y_{k}$. La rétraction $R_{J}$ est définie sur un polydisque fibré dans $N_{Y_{J} \mid X}$ à valeurs dans $N_{Y_{J} \mid X} \simeq \times_{i \in J}\left(N_{Y_{i} \mid X} \mid Y_{J}\right)$ 


\section{B. Audoubert, F. El Zein et Lê Dũng Tráng}

et les morphismes de recollement par les matrices diagonales unitaires opèrent sur la source et le but de $R_{J}$ de manière compatible avec $R_{J}$; elle s'identifie via le plombage à une rétraction $R_{J}: U_{J} \longrightarrow \bigcup_{k} Y_{k}$ d'un voisinage $U_{J}$ de $Y_{J}$ dans $X$ sur la réunion des hyperplans $\bigcup_{k} Y_{k}$. Les différentes restrictions des projections $R_{J}$ à $\overline{U_{J}} \backslash\left(Y_{J}\left(K_{*}\right) \cup \hat{U}_{J}\right)$, où $\hat{U}_{J}=\bigcup_{L, J \subset L} U_{L}=U_{J} \cap\left(\bigcup_{i \notin J} U_{i}\right)$, se recollent bien car pour $J \subset L, R_{L}$ et $R_{J}$ coïncident sur le bord $\partial\left(\overline{U_{L}} \backslash\left(Y_{J}\left(K_{*}\right) \cup \hat{U}_{L}\right)\right)$. La projection $R$ est en fait la restriction d'une rétraction continue différentiable par morceaux.

\subsection{Rétraction de la fibre de Milnor sur le DCN}

La fibre de Milnor se trouvant pour $|t|$ assez petit dans un voisinage régulier $U$, la rétraction $R$ de $U$ sur $Y$ induit une projection stratifiée $\Pi: F_{t} \longrightarrow Y$ qui envoie $F_{t, J}$ sur $Y_{J}$.

Localement dans la résolution $Z$, la fibre de Milnor est définie par une équation monomiale $a \prod_{i=1}^{n} z_{i}^{n_{i}}=t$, où $a$ est un nombre complexe non nul. Nous aurons besoin de la transformation introduite dans l'étude locale suivante.

3.3.1 Etude locale de la fibre de Milnor. On considère la fibre $F_{t}$ définie dans $\mathbb{C}^{n}$ par l'équation

$$
a \prod_{i=1}^{n} z_{i}^{n_{i}}=t
$$

où $a$ est un nombre complexe. En fait on ne considèrera que l'intersection de cette fibre avec le polydisque où tous les rayons $K_{i}$ sont égaux à un nombre fixé $K$ et où $|t|$ est assez petit, e.g. $K>(|t| /|a|)^{\left(1 / \Sigma n_{i}\right)}$. On introduit la fibre réelle $F_{|t|}^{\prime}$ définie par l'équation $|a| \prod_{i=1}^{n} t_{i}^{\prime n_{i}}=|t|$, avec $t_{i}^{\prime}<K$. L'application induite par la norme sur les fibres de Milnor

$$
N: F_{t} \longrightarrow F^{\prime}{ }_{|t|}: N\left(z_{1}, \ldots, z_{n}\right)=\left(\left|z_{1}\right|, \ldots,\left|z_{n}\right|\right)
$$

est fibrée en une réunion de disjointe de $d=\operatorname{pgcd}\left\{n_{1}, \ldots, n_{n}\right\}$ tores de dimension $n-1$. Si on écrit $z_{j}=\rho_{j} e^{i \theta_{j}}, t=\rho e^{i \theta}$ où $\theta, \theta_{j} \in \mathbb{Z} / 2 \pi \mathbb{Z}$, une fibre de $N$ est définie par la condition $\sum_{i=1}^{n} n_{i} \theta_{i}=\theta+2 k \pi$, ce qui fait que les tores sont naturellement plongés dans un tore distingué de $\mathbb{C}^{n}$ de dimension $n$; $N$ est une fibration trivialisable.

Un moyen de simplifier les calculs est d'introduire la transformation

$$
T:\left(\mathbb{R}^{+}\right)^{n} \longrightarrow \mathbb{R}^{n}
$$

où les coordonnées de $T$ sont les $s_{i}=T_{i}\left(t_{1}^{\prime}, \ldots, t_{n}^{\prime}\right)=\log K-\log t_{i}^{\prime}$. Alors $T\left(F_{|t|}^{\prime}\right)$ est l'hyperplan $H$ défini par l'équation

$$
\sum_{i=1}^{n} n_{i} s_{i}=\left(\sum_{i=1}^{n} n_{i}\right) \log K+\log |a|-\log |t|=A^{\prime}-\log |t|
$$

où l'on a posé $A^{\prime}=\left(\sum_{i=1}^{n} n_{i}\right) \log K+\log |a|$.

Une face définie par $t_{i}^{\prime}=K$ correspond à l'hyperplan défini par $s_{i}=0$ et $t_{i}^{\prime} \leqslant K$ est équivalent à $s_{i} \geqslant 0\left(|t|\right.$ assez petit) de sorte que la partie de la fibre réelle $F_{|t|}^{\prime}$ située dans le polycube $P^{\prime}(K)$ de côtés $K$ se transforme en un simplexe $\Delta_{n-1}=H \cap\left(\mathbb{R}^{+}\right)^{n}$ de sommets $\left(0, \ldots,\left(1 / n_{i}\right)\left(A^{\prime}-\right.\right.$ $\log |t|), \ldots, 0)$.

On introduit la stratification suivante de $F_{|t|}^{\prime}$ donnée par les strates $F_{|t|}^{\prime} \cap Q_{J}^{\prime}$, pour tout $J \subset[1, n]$,

$$
F_{|t|, J}^{\prime}=\left\{\left(t_{1}^{\prime}, \ldots, t_{n}^{\prime}\right) \in F_{t^{\prime}}^{\prime}: t_{i}^{\prime}=t_{j}^{\prime}, i, j \in J, t_{i}^{\prime}<t_{j}^{\prime}, i \in J, j \notin J\right\} .
$$

Remarquons que cette stratification sur $F_{|t|}^{\prime} \cap P^{\prime}(K)$ correspond à la décomposition barycentrique du simplexe $\Delta_{n-1}$. 


\section{INVARIANTS D'UNE DÉSINGULARISATION ET SINGULARITÉS DES MORPHISMES}

Dans le cas complexe, la stratification de $F_{t}$ est donnée par les

$$
F_{t, J}=\left\{\left(z_{1}, \ldots, z_{n}\right) \in F_{t}:\left|z_{i}\right|=\left|z_{j}\right|, i, j \in J,\left|z_{i}\right|<\left|z_{j}\right|, i \in J, j \notin J\right\} .
$$

Elle s'envoie sur $Y_{J}$ par la projection $\Pi$.

3.3.2 Cas général. Ce cas reprend l'étude locale et la généralise au cas d'un système de fibrés normaux, utilisant la technique de plombage. Il ressemble mot pour mot au cas général déjà traité ci-dessus.

\section{Démonstration du théorème 1}

En fait nous démontrons d'abord le lemme suivant où le nombre $B$ a été introduit au $\S 2.3$ :

Lemme 1. Pour un choix convenable du nombre $B$, la filtration polaire est incluse dans la filtration d'Hironaka $F_{t}(r) \subset F_{t}^{H}(r)$, ce qui signifie que l'on a l'inclusion

$$
F_{t}(r)=g^{-1}\left(D\left(0, B|t|^{r}\right)\right) \cap F_{t} \subset U(r):=\bigcup_{i \in I^{\prime}, q(i) \geqslant r} U_{i} .
$$

De plus le bord de la filtration polaire vérifie

$$
\partial F_{t}(r) \subset V(r):=\bigcup_{i \in I^{\prime}, q(i) \geqslant r, q(j)<r}\left(U_{i} \cap U_{j}\right) .
$$

Démonstration. Revenons aux notations du théorème 1 ; en particulier le DCN est de nouveau noté $D$ et les fonctions induites sur la désingularisée $Z$ sont toujours notées $f$ et $g$.

Soit $J \subset I^{\prime}$ une partie de longueur $|J|=p$ qui rencontre $I$. D'après Clemens (voir théorème 2, partie vi, § 3.1) il existe des coordonnées locales $\left(y, v_{1}, \ldots, v_{p}\right)$ de $N_{D_{J} \mid Z}$ telles que $f$ s'écrive

$$
f=c_{f}(y) v_{1}^{n_{1}} \cdots v_{p}^{n_{p}}
$$

où $c_{f}(y)$ est une fonction inversible différentiable en les coordonnées $y$ sur $D_{J}$. Par ailleurs quand $a$ est un point de $D_{J}$, la restriction de la fonction $g: Z \longrightarrow \mathbf{C}$ à la fibre $N_{D_{J} \mid Z}(a)$ s'écrit

$$
g=h(a, v) v_{1}^{m_{1}} \cdots v_{p}^{m_{p}}
$$

où $m_{i}$ est la multiplicité de $g$ le long de $D_{i}, 1 \leqslant i \leqslant p$, et $h(a, v)$ une fonction holomorphe en $v$ et inversible. Clemens (voir théorème $2, \S 3.1$ ) donne une description d'un voisinage régulier de $D_{J}$ comme une famille de fibres holomorphes paramétrées par les points $a \in D_{J}$, variant de manière différentiable avec $a$, ce qui assure pour les coordonnées $(y, v)$ l'existence d'une fonction inversible $h(y, v)$, différentiable en $y$ et holomorphe en $v$, telle que $g$ s'écrive

$$
g=h(y, v) v_{1}^{m_{1}} \cdots v_{p}^{m_{p}} .
$$

La fibre de Milnor $F_{t}$ est définie par l'équation

$$
c_{f}(y) v_{1}^{n_{1}} \cdots v_{p}^{n_{p}}=t
$$

et la sous-variété polaire $F_{t}(r)$ par l'inégalité

$$
\left|h(y, v) v_{1}^{m_{1}} \cdots v_{p}^{m_{p}}\right|<B|t|^{r} .
$$

En combinant l'égalité et l'inégalité on trouve

$$
\left|h(y, v) v_{1}^{m_{1}-r n_{1}} \cdots v_{p}^{m_{p}-r n_{p}}\right|<B\left|c_{f}(y)\right|^{r}
$$

et en prenant le logarithme on trouve

$$
\log |h(y, v)|+\sum_{j \in J}\left(m_{j}-r n_{j}\right) \log \left|v_{j}\right|<\log B+r \log \left|c_{f}(y)\right| .
$$




\section{B. Audoubert, F. El Zein et Lê Dũng Tráng}

Dans le cas où pour tout $j \in J$ les quotients d'Hironaka $q(j)$ sont $\geqslant r$, on a $m_{j}-r n_{j} \geqslant 0$ et l'inégalité (1) a toujours lieu quand $B$ est fixé assez grand et $|t|$ assez petit. Alors que dans le cas où pour tout $j \in J$ les quotients d'Hironaka $q(j)$ sont $<r$, on a $m_{j}-r n_{j}<0$ et l'inégalité (1) ne peut jamais avoir lieu pour tout choix de $B$ fixé, lorsque $|t|$ devient assez petit.

Par conséquent, là où $q(j) \geqslant r$ pour tout $j \in J$, les termes des deux filtrations coïncident, ce qui montre la première inclusion et ils sont totalement disjoints là où $q(j)<r$ pour tout $j \in J$, ce qui montre la deuxième inclusion portant sur le bord $\partial F_{t}(r)$ de la filtration polaire, donnée en coordonnées locales par l'égalité $\left|h(y, v) v_{1}^{m_{1}} \cdots v_{p}^{m_{p}}\right|=B|t|^{r}$.

Remarque. Le fait que la filtration polaire $F_{t}(r)$ et la filtration d'Hironaka $F_{t}^{H}(r)$ coïncident exactement en dehors d'un collier du bord de la filtration polaire montre que, pour démontrer le théorème, il suffit de construire un difféomorphisme au voisinage de ce bord, i.e. dans le voisinage $V(r)$, que l'on prolonge ensuite par l'identité.

On démontre d'abord le lemme suivant.

Lemme 2 (Changement de variables). Soient $D=\bigcup_{1 \leqslant i \leqslant e} D_{i}$ un $D C N$ dans un polydisque de $\mathbb{C}^{e}$, $f$ et $g$ deux fonctions holomorphes telles que leur diviseur $(f)=\sum_{i=1}^{e} n_{i} D_{i}$ et $(g)=\sum_{i=1}^{e} m_{i} D_{i}$ soient non proportionnels; alors on peut trouver des coordonnées $x_{i}, i=1, \ldots, e$ de $\mathbb{C}^{e}$ telles que l'on ait simultanément $f=\prod_{i=1}^{e} x_{i}^{n_{i}}$ et $g=\prod_{i=1}^{e} x_{i}^{m_{i}}$.

Démonstration. Soient $y_{i}, i=1, \ldots, e$, les coordonnées du polydisque. Les décompositions en éléments irréductibles s'écrivent par hypothèse $f=h \prod_{i} y_{i}^{n_{i}}$ et $g=h^{\prime} \prod_{i} y_{i}^{m_{i}}$ où $h$ et $h^{\prime}$ sont analytiques inversibles et les quotients $m_{i} / n_{i}$ non tous égaux. On considère le changement de variables défini par

$$
x_{i}=\varphi_{i}(y .)=\rho_{i}(y \cdot) y_{i}, \quad \text { où } \varphi=\left(\varphi_{1}, \ldots, \varphi_{e}\right):\left(\mathbb{C}^{e}, 0\right) \longrightarrow\left(\mathbb{C}^{e}, 0\right) .
$$

Si on suppose $n_{1} / m_{1} \neq n_{2} / m_{2}$, on pose $\rho_{i}=1$ pour $i>2$. La différentielle $d_{0} \varphi$ de $\varphi$ s'écrit

$$
\begin{aligned}
& \partial \varphi_{1} / \partial y_{1}=y_{1} \partial \rho_{1} / \partial y_{1}+\rho_{1}, \quad \partial \varphi_{1} / \partial y_{2}=y_{1} \partial \rho_{1} / \partial y_{2}, \\
& \partial \varphi_{2} / \partial y_{1}=y_{2} \partial \rho_{2} / \partial y_{1}, \quad \partial \varphi_{2} / \partial y_{2}=y_{2} \partial \rho_{2} / \partial y_{2}+\rho_{2},
\end{aligned}
$$

$\partial \varphi_{3} / \partial y_{i}=0$, sauf pour $i=3$ c'est égal à 1 , et ainsi de suite. On calcule

$$
\operatorname{dét}\left(d_{0} \varphi\right)=\left[\left(y_{2} \frac{\partial \rho_{2}}{\partial y_{2}}+\rho_{2}\right)\left(y_{1} \frac{\partial \rho_{1}}{\partial y_{1}}+\rho_{1}\right)-\left(y_{1} \frac{\partial \rho_{1}}{\partial y_{2}}\right)\left(y_{2} \frac{\partial \rho_{2}}{\partial y_{1}}\right)\right](0)=\rho_{1} \rho_{2}(0) \text {. }
$$

On cherche $\rho_{1}$ et $\rho_{2}$ tel que

1) $\rho_{1}^{n_{1}} \rho_{2}^{n_{2}}=h$;

2) $\rho_{1}^{m_{1}} \rho_{2}^{m_{2}}=h^{\prime}$

3) $\rho_{1} \rho_{2}(0) \neq 0$.

En éliminant $\rho_{2}$, on trouve $\rho_{1}^{m_{1} n_{2}-n_{1} m_{2}}=h^{\prime n_{2}} h^{-m_{2}}$, soit

$$
\rho_{1}=h^{\prime-n_{2} /\left(m_{1} n_{2}-n_{1} m_{2}\right)} h^{-m_{2} /\left(m_{1} n_{2}-n_{1} m_{2}\right)}
$$

ce qui a un sens car $h^{\prime}$ et $h$ sont analytiques inversibles et précisément $m_{1} n_{2}-n_{1} m_{2} \neq 0$. On en déduit $\rho_{2}$.

Démonstration de la proposition 1 et du théorème 1.

i) Construction du difféomorphisme de la filtration d'Hironaka à la filtration polaire, noté $\varphi_{J}$ audessus d'une composante $D_{J}^{\star}$.

Pour toute partie $J \subset I^{\prime}$ de longueur $|J|=p$ contenant deux indices distincts $i$ et $j$ tels que $q(i) \neq q(j)$, on peut trouver d'après le lemme 2 , au voisinage de tout point général de $D_{J}^{\star}$, 
des coordonnées $z_{1}, \ldots, z_{p}$ faisant partie d'un système de coordonnées locales de la désingularisée $Z$ telles que $f=\prod_{i \in J} z_{i}^{n_{i}}$ et $g=\prod_{i \in J} z_{i}^{m_{i}}$. D'après Clemens (voir théorème 2, partie vi, $\S 3.1$ ) on peut faire un nouveau changement de variables pour écrire $f$ et $g$ sous la forme

$$
f=c_{f}(y) \prod_{i \in J} v_{i}^{n_{i}}, g=c_{g}(y) \prod_{i \in J} v_{i}^{m_{i}}
$$

avec $\left(y, v_{1}, \ldots, v_{p}\right)$ des coordonnées de $N_{D_{J} \mid Z}$ où les $v_{i}$ sont linéaires sur les fibres et les fonctions $c_{f}(y)$ et $c_{g}(y)$ différentiables en les coordonnées $y$ dans $D_{J}$. On suppose la décomposition $N_{D_{J} \mid Z} \simeq$ $\prod_{i \in J}\left(N_{D_{i} \mid Z} \mid D_{J}\right)$ orthogonale et on considère l'application norme

$$
N: N_{D_{J} \mid Z} \longrightarrow D_{J} \times\left(\mathbb{R}^{+}\right)^{p}: N\left(y, v_{i_{1}}, \ldots, v_{i_{p}}\right)=\left(y,\left|v_{i_{1}}\right|, \ldots,\left|v_{i_{p}}\right|\right) .
$$

Indifféremment on note aussi $N$ la norme définie sur un voisinage régulier $U_{J}$ de $D_{J}$ ou son adhérence ; cette norme $N$ est bien définie à valeurs dans un fibré trivial, car les changements de coordonnées dans le fibré normal sont définis par des matrices diagonales unitaires.

Le bord de la filtration d'Hironaka fait éventuellement partie du bord de

$$
N^{-1}\left(D_{J} \times P_{J}(K)\right) \cap F_{t}
$$

où $P_{J}(K)$ est fibré sur $D_{J}$ en polycubes de rayon $K$ alors que le bord de la filtration polaire est déterminé par des relations du type

$$
\left|g\left(y, v_{i_{1}}, \ldots, v_{i_{p}}\right)\right|<B|t|^{r} \quad \text { soit }\left|c_{g}(y)\right| \prod_{i \in J}\left|v_{i}\right|^{m_{i}}<B|t|^{r},
$$

autrement dit c'est aussi une inégalité portant sur l'image par $N$.

Dans la suite on considère un voisinage régulier $U_{J}$ de $D_{J}$ formé d'un fibré au-dessus de $D_{J}$ en polydisque de rayons $K$, d'adhérence $\overline{U_{J}}$ que l'on suppose plongé dans $N_{D_{J} \mid Z}$ et un point $a$ en position générale, au sens que $a \in D_{J}^{\star}$ où $D_{J}^{\star}=D_{J} \backslash \bigcup_{i \in I^{\prime} \backslash J} D_{i}$. La fibre de Milnor $F_{t}$ de $f$ est définie par l' équation $c_{f}(a) \prod_{i \in J} v_{i}^{n_{i}}=t$, avec $\left|v_{i}\right|<K, K$ assez grand et son image par la norme $F_{|t|}^{\prime}$ par l'équation $\left|c_{f}(a)\right| \prod_{i \in J}\left|v_{i}\right|^{n_{i}}=|t|$, avec $\left|v_{i}\right|<K$.

Soit $T$ la transformation (décrite en $\S 3.3$ ) définie par le changement de variables $\log \left|v_{i}\right|=$ $\log K-s_{i}$. L'hyperplan défini par $\left|v_{i}\right|=K$ correspond à l'hyperplan défini par $s_{i}=0$. L'équation de $F_{|t|}^{\prime}$ devient l'équation d'un hyperplan réel $H$ défini par

$$
\sum_{i \in J} n_{i} s_{i}=\left(\sum_{i \in J} n_{i}\right) \log K+\log \left|c_{f}(a)\right|-\log |t|=A^{\prime}-\log |t|
$$

où l'on a posé $A^{\prime}=\left(\sum_{i \in J} n_{i}\right) \log K+\log \left|c_{f}(a)\right|$. La partie de $F_{|t|}^{\prime}$ située dans le fibré en polycubes $P(K)$ de côtés $K$ se transforme donc en un simplexe $\Delta_{p-1}=H \cap\left(\mathbb{R}^{+}\right)^{p}$ de sommets

$$
\left(0, \ldots,\left(1 / n_{i}\right)\left(A^{\prime}-\log |t|\right), \ldots, 0\right) .
$$

L'inégalité $\left|c_{g}(a)\right| \prod_{i \in J}\left|v_{i}\right|^{m_{i}}<B|t|^{r}$ se transforme par Log en

$$
\log \left|c_{g}(a)\right|+\sum_{i \in J} m_{i} \log \left|v_{i}\right|<\log B+r \log |t|
$$

ce qui donne après transformation par $T$

$$
\log \left|c_{g}(a)\right|+\sum_{i \in J} m_{i} \log K-\sum_{i \in J} m_{i} s_{i}<\log B+r \log |t|
$$

soit

$$
\sum_{i \in J} m_{i} s_{i}>A^{\prime \prime}-\log B-r \log |t|
$$




\section{B. Audoubert, F. El Zein et Lê Dũng Tráng}

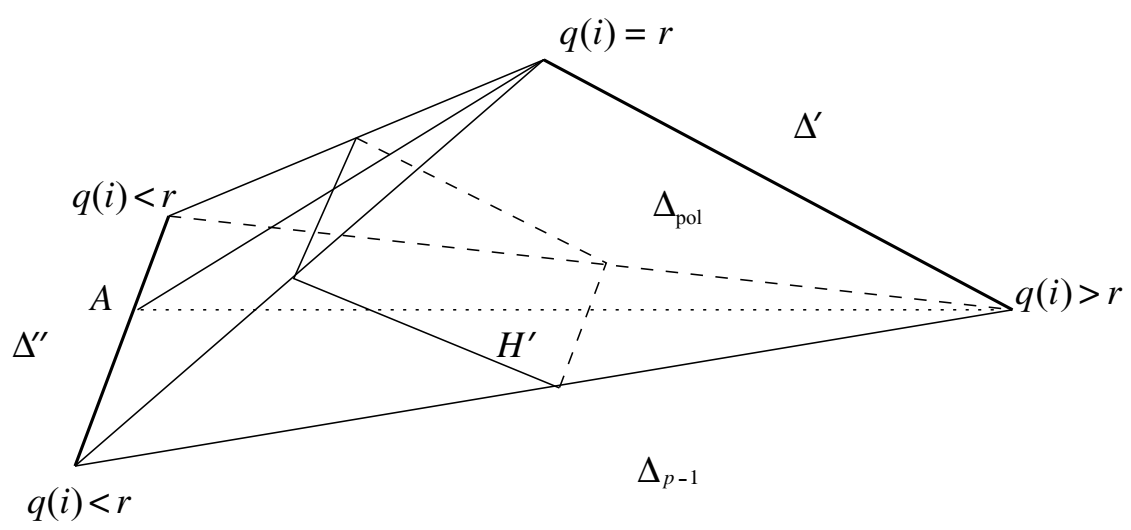

FIGURE 1.

où $A^{\prime \prime}=\log \left|c_{g}(a)\right|+\sum_{i \in J} m_{i} \log K$. C'est une inégalité définie par un hyperplan $H^{\prime}$. Un sommet

$$
\left(0, \ldots, s_{k}, \ldots, 0\right)=\left(0, \ldots,\left(1 / n_{k}\right)\left(A^{\prime}-\log |t|\right), \ldots, 0\right)
$$

satisfait cette inégalité si et seulement si

$$
\left(m_{k} / n_{k}\right)\left(A^{\prime}-\log |t|\right)>A^{\prime}-\log B-r \log |t|
$$

soit

$$
\left(m_{k} / n_{k}\right) A^{\prime}-A^{\prime}+\log B>\left(\left(m_{k} / n_{k}\right)-r\right) \log |t| .
$$

Ceci est assuré pour $t$ fixé si $B$ est assez grand et pour $|t|$ assez petit si et seulement si $m_{k} / n_{k} \geqslant r$. On peut aussi faire $(3)-r(2)$, ce qui donne une inégalité indépendante de $t$

$$
\sum_{i \in J}\left(m_{i}-r n_{i}\right) s_{i} \geqslant A^{\prime \prime}-r A^{\prime}-\log B
$$

définie par un hyperplan qui rencontre l'axe de coordonnée $s_{k}$ pour la valeur de $s_{k}$ satisfaisant l'égalité

$$
\left(m_{k}-r n_{k}\right) s_{k}=A^{\prime \prime}-r A^{\prime}-\log B .
$$

Rappelons que l'on a transformé ainsi la fibre de Milnor réelle contenue dans un polycube, fibre au-dessus d'un point $a$ de $D_{J}^{\star}$, en un simplexe $\Delta_{p-1}$. L'hyperplan $H^{\prime}$ coupe ce simplexe $\Delta_{p-1}$ en deux parties, l'une polaire que l'on désigne par $\Delta_{\text {pol }}$ contenant un sous-simplexe $\Delta^{\prime}$ engendré par les sommets de quotient d'Hironaka $q(i) \geqslant r$ et correspondant à la filtration polaire, l'autre contenant un sous-simplexe $\Delta^{\prime \prime}$ engendré par les sommets de quotient d'Hironaka $q(i)<r$ (voir la figure 1).

Le simplexe $\Delta_{p-1}$ est le joint de ces deux sous-simplexes, c'est-à-dire la réunion des segments joignant deux points de ces sous-simplexes. Dans ces conditions on peut construire un difféomorphisme naturel $\varphi_{a}^{\prime}$ le long de ces segments, transformant $\Delta_{p-1} \backslash \Delta^{\prime \prime}$ en $\Delta_{\text {pol }} \backslash H^{\prime}$ et induisant l'identité près de $\Delta^{\prime}$. Ce résultat est clair au niveau des simplexes réels, on peut cependant donner les indications suivantes. L'hyperplan $H^{\prime}$ est défini par une équation linéaire $l=0$. On peut construire une fonction rélle sur le simplexe $\Delta_{p-1}$ naturellement stratifiée de fibre $\Delta^{\prime \prime}$ en un point $\alpha>0$, l'hyperplan $H^{\prime}$ en 0 et sans point critique dans la région $\Delta_{p-1} \backslash\left(\Delta^{\prime \prime} \cup \Delta_{\text {pol }}\right)$ au-dessus de l'intervalle ouvert $] 0, \alpha[$. Par un argument de théorie de Morse, on peut construire le difféomorphisme cherché en utilisant un champ de vecteurs.

On peut cependant construire directement un champ de vecteurs $C^{\infty}$ sur $\Delta_{p-1} \backslash \Delta^{\prime \prime}$ qui s'annule sur un voisinage de $\Delta^{\prime}$ comme suit. Pour tout point $A \in \Delta^{\prime \prime}$, on considère l'ensemble $C_{A}\left(\Delta^{\prime}\right)$ des droites affines qui joignent le point $A$ à un point de $\Delta^{\prime}$. Soit $D \in C_{A}\left(\Delta^{\prime}\right)$ une telle droite. On choisit 


\section{INVARIANTS D'UNE DÉSINGULARISATION ET SINGULARITÉS DES MORPHISMES}

sur la droite $D$ un champ de vecteurs $\xi_{D}$ qui s'annule sur un voisinage de $D \cap \Delta^{\prime}$ et dont le flot au temps 1 envoie le point $A$ sur le point $D \cap H^{\prime}$. On définit alors le champ de vecteur $\xi$ que l'on cherche sur $\Delta_{p-1} \backslash \Delta^{\prime \prime}$ (ne contenant pas le point $A$ ) en exigeant que, pour tout point $A \in \Delta^{\prime \prime}$ et pour toute droite affine $D \in C_{A}\left(\Delta^{\prime}\right)$, la restriction de $\xi$ à $D \cap\left(\Delta_{p-1} \backslash \Delta^{\prime \prime}\right)$ coïncide avec le champ $\xi_{D}$ défini ci-dessus. On parle d'un champ naturel dans le sens où la restriction du champ aux faces du simplexe coïncide avec le champ construit sur le simplexe en dimension inférieure. Le difféomorphisme $\varphi_{a}^{\prime}$ est le flot au temps $1 \mathrm{du}$ champ de vecteurs $\xi$.

L'intersection de la fibre de Milnor complexe avec le polydisque complexe est, via l'application norme $N$, un fibré en tores de dimension $p-1$, trivial sur le simplexe $\Delta_{p-1}$, ce qui permet de relever $\varphi_{a}^{\prime}$ en un difféomorphisme $\varphi_{a}$ sur cette intersection. Lorsque le point $a$ varie sur $D_{J}^{\star}$, le difféomorphisme $\varphi_{a}^{\prime}$ sur la fibre réelle au-dessus de $a$, détermine un difféomorphisme $\varphi_{J}^{\prime}$ sur la fibre réelle dans le fibré trivial $D_{J}^{\star} \times\left(\mathbb{R}^{+}\right)^{p}$, alors que les difféomorphismes $\varphi_{a}$ sur la fibre complexe audessus de $a$ se recollent en un difféomorphisme $\varphi_{J}$ ainsi défini. Si $N$ est considéré sur $\overline{U_{J}} \subset N_{D_{J} \mid Z}$, on a en posant $N_{T}=T \circ N$, où $T$ a été définie au $\S 3.3$, à valeurs dans le fibré trivial de simplexes,

$$
\overline{U_{J}} \cap F_{t} \cap \Pi_{J}^{-1}\left(D_{J}^{\star}\right) \backslash N_{T}^{-1}\left(\Delta_{J}^{\prime \prime}\right) \stackrel{\varphi_{J}}{\longrightarrow} \overline{U_{J}} \cap F_{t} \cap \Pi_{J}^{-1}\left(D_{J}^{\star}\right) \backslash N_{T}^{-1}\left(\left(\Delta_{\mathrm{pol}} \backslash H^{\prime}\right)_{J}\right)
$$

où $\Delta_{J}^{\prime \prime}=\bigcup_{a \in D_{J}^{\star}}\{a\} \times \Delta^{\prime \prime}$ et $\left(\Delta_{\text {pol }} \backslash H^{\prime}\right)_{J}=\bigcup_{a \in D_{J}^{\star}}\{a\} \times\left(\Delta_{\text {pol }} \backslash H^{\prime}\right)$. Précisément le difféomorphisme $\varphi_{J}$ sur $\overline{U_{J}} \cap F_{t} \cap \Pi_{J}^{-1}\left(D_{J}^{\star}\right) \backslash N_{T}^{-1}\left(\Delta_{J}^{\prime \prime}\right)$ est défini comme suit : sur une fibre au-dessus d'un point $a \in D_{J}^{\star}$ de coordonnées

$$
t_{j .}=\left(t_{j_{1}}, \ldots, t_{j_{k}}, \ldots, t_{j_{p}}\right)=\left(\left|t_{j_{1}}\right| e^{i \theta_{j_{1}}}, \ldots,\left|t_{j_{k}}\right| e^{i \theta_{j_{k}}}, \ldots,\left|t_{j_{p}}\right| e^{i \theta_{j_{p}}}\right)
$$

on pose

$$
\varphi_{J}\left(a, t_{j .}\right)=\left(\varphi_{J, j_{1}}^{\prime}\left(a,\left|t_{j_{1}}\right|, \ldots,\left|t_{j_{p}}\right|\right) e^{i \theta_{j_{1}}}, \ldots, \varphi_{J, j_{1}}^{\prime}\left(a,\left|t_{j_{1}}\right|, \ldots,\left|t_{j_{p}}\right|\right) e^{i \theta_{j_{p}}}\right)
$$

où $\varphi_{J, j_{k}}^{\prime}$ désigne la composante d'indice $j_{k} \in J$ du difféomorphisme réel $\varphi_{J}^{\prime}$ défini ci-dessus. Cette définition est compatible avec les morphismes de recollement dans le fibré normal.

ii) Construction du difféomorphisme global $\varphi$.

Aussi les différents difféomorphismes construits lorsque $J$ varie se recollent pour définir un difféomorphisme $\varphi$ au voisinage de tous les $D_{J}$ avec deux quotients d'Hironaka distincts séparés par $r$ (rappelons que l'on a $q(i) \geqslant r>q(j)$, là où le bord de la filtration polaire se trouve). Sur la réunion des ouverts $U_{J}$ avec deux quotients distincts séparés par $r(q(i) \geqslant r>q(j))$ on construit le difféomorphisme $\varphi$ comme suit. On prend pour tout $J$ la restriction de $\varphi_{J}$ à

$$
\left(\overline{U_{J}} \backslash \hat{U}_{J}\right) \cap F_{t} \backslash N_{T}^{-1}\left(\Delta_{J}^{\prime \prime}\right) \text { où } \hat{U}_{J}=\bigcup_{L, J \subset L} U_{L}=U_{J} \cap\left(\bigcup_{i \notin J} U_{i}\right) \text {. }
$$

Les différentes restrictions des difféomorphismes $\varphi_{J}$ se recollent bien car pour $J \subset L, \varphi_{L}$ et $\varphi_{J}$ coïncident sur le bord $\partial\left(\overline{U_{L}} \backslash N_{T}^{-1}\left(\Delta_{L}^{\prime \prime}\right) \backslash \hat{U}_{L}\right) \cap F_{t}$. Le difféomorphisme $\varphi$ est en fait différentiable par morceaux.

iii) Relation avec la projection stratifiée $\Pi$. Construction du difféomorphisme sur $\Pi^{-1}\left(D^{\star}(r)\right) \cup$ $F_{t}^{\infty}(r)$.

La sous-variété d'Hironaka $F_{t}^{H}(r)$ et la sous-variété associée à la projection stratifiée $\Pi^{-1}\left(D^{\star}(r)\right)$ $\cup F_{t}^{\infty}(r)$ coïncident en dehors de l'ouvert $\bigcup_{i \in I, q(i) \geqslant r, q(j)<r} U_{i} \cap U_{j}$, dans lequel on va construire un difféomorphisme par morceaux, étendu par l'identité. La même étude qu'en (i) nous ramène à regarder le difféomorphisme restreint à $N_{T}^{-1} \Delta_{p-1}$. La projection $\Pi$ envoie l'inverse par $N_{T}$ de la subdivision barycentrique sur la réunion des hyperplans, d'où l'image réciproque de $D(r)$ est l'inverse par $N_{T}$ de l'étoile $\Delta^{\prime}(\star)$ du sous-simplexe $\Delta^{\prime}$ dans la subdivision barycentrique de $\Delta_{p-1}$. A ce stade on construit ce nouveau difféomorphisme de manière identique au précédent, i.e. que l'on est ramené à construire un difféomorphisme par morceaux naturel de $\Delta_{p-1} \backslash \Delta^{\prime \prime} \operatorname{sur} \Delta^{\prime}(\star) \backslash \partial \Delta^{\prime}(\star)$, 


\section{B. Audoubert, F. El Zein et Lê Dũng Tráng}

qui coïncide avec l'identité sur un voisinage de $\Delta^{\prime}$. Pour tout point $A \in \Delta^{\prime \prime}$, on considère le cône $C_{A}\left(\Delta^{\prime}\right)$ des droites affines qui joignent le point $A$ à un point de $\Delta^{\prime}$. Soit $D \in C_{A}\left(\Delta^{\prime}\right)$ une telle droite. On choisit sur la droite $D$ un champ de vecteurs $\xi_{D}$ qui s'annule sur un voisinage de $D \cap \Delta^{\prime}$ et dont le flot au temps 1 envoie le point $A$ sur le point $D \cap \partial \Delta^{\prime}(\star)$. On définit alors un champ de vecteur $\xi$ différentiable par morceaux sur $\Delta_{p-1} \backslash \Delta^{\prime \prime}$ en exigeant que, pour tout point $A \in \Delta^{\prime \prime}$ et pour toute droite affine $D \in C_{A}\left(\Delta^{\prime}\right)$, la restriction de $\xi$ à $D$ coïncide avec le champ $\xi_{D}$. Le difféomorphisme par morceaux que l'on veut est le flot au temps 1 du champ $\xi$.

iv) Cas de la couronne polaire : démonstration de l'assertion ii) de la proposition 1.

La condition portant sur la couronne, soit $A|t|^{r}<|g|<B|t|^{r}$, s'obtient en rajoutant à la condition précédente étudiée ci-dessus une condition qui lui est duale, ce qui fait que les raisonnements qui suivent seront analogues aux précédents. On a les inclusions

$$
|g|^{-1}(] A|t|^{r},+\infty[) \cap F_{t} \subset \bigcup_{i \in I^{\prime}, q(i) \leqslant r} U_{i}
$$

et

$$
|g|^{-1}\left(\left[0, B|t|^{r}[) \cap F_{t} \subset \bigcup_{i \in I^{\prime}, q(i) \geqslant r} U_{i}\right.\right.
$$

D'où

$$
g^{-1}(C(r)) \cap F_{t} \subset\left(\bigcup_{q(i)=r} U_{i}\right) \cup\left(\bigcup_{q(i)>r, q(j)<r} U_{i} \cap U_{j}\right) .
$$

Nous allons montrer qu'il existe une isotopie stratifiée entre

$$
g^{-1}(C(r)) \cap F_{t} \quad \text { et } \quad F_{t} \cap\left(\bigcup_{q(i)=r} U_{i} \cup\left(\bigcup_{q(i)>r, q(j)<r} U_{i} \cap U_{j}\right)\right) .
$$

Par la technique de plombage de Clemens, l'étude globale peut être ramenée à l'étude de la fibre de Milnor $c_{f}(a) v_{1}^{n_{1}} \ldots v_{p}^{n_{p}}=t$ dans un polydisque d'une fibre $N_{D_{J} \mid Z}(a)$ du fibré normal (où on suppose $|J|=p$ et qu'il existe $i, j \in J$ tel que $q(j)<r<q(i))$. La couronne polaire est déterminée par la double inégalité

$$
A|t|^{r}<\left|c_{g}(a) v_{1}^{m_{1}} \cdots v_{p}^{m_{p}}\right|<B|t|^{r} .
$$

Par le changement de coordonnées $T$, la fibre de Milnor devient un simplexe $\Delta_{p-1}$ coupé par deux hyperplans $H^{\prime}$ (transformé de l'hypersurface réelle $\left|c_{g}(a) v_{1}^{m_{1}} \cdots v_{p}^{m_{p}}\right|=B|t|^{r}$ ) et $H^{\prime \prime}$ (transformé de l'hypersurface $\left.\left|c_{g}(a) v_{1}^{m_{1}} \cdots v_{p}^{m_{p}}\right|=A|t|^{r}\right)$, qui délimitent une zone $\Delta(r)$ correspondant à la couronne polaire.

On introduit les sous-simplexes $\Delta^{\prime}$ (respectivement $\Delta^{\prime \prime}$ et $\Delta^{\prime \prime \prime}$ ) engendrés par les sommets de quotient d'Hironaka $q(i)>r$ (respectivement $q(i)<r$ et $q(i)=r$ ), formant ainsi une partition de $\Delta_{p-1}$. Désignons par $\Delta(\star)$ l'étoile d'un sous-simplexe $\Delta$ de $\Delta_{p-1}$. Pour un choix des nombres $A$ et $B$ convenables, on peut supposer que l'hyperplan $H^{\prime}$ délimite une région $\Delta_{\text {pol }}$ contenant $\Delta^{\prime}(\star) \cup \Delta^{\prime \prime \prime}(\star)$ et que l'hyperplan $H^{\prime \prime}$ délimite une région $\Delta_{\text {apol }}$ contenant $\Delta^{\prime \prime}(\star) \cup \Delta^{\prime \prime \prime}(\star)$ (voir la figure 2). Dans ce cas on doit construire un difféomorphisme de chaque côté par exemple à l'aide d'une théorie de Morse convenable des deux côtés, ou en adaptant la construction précédente du champ de vecteurs. On considère un voisinage $U^{\prime}$ de $\Delta^{\prime}(\star) \cup \Delta^{\prime \prime \prime}(\star)$ dans $\Delta_{\text {pol }} \subset \Delta_{p-1} \backslash \Delta^{\prime \prime}$ et un voisinage $U^{\prime \prime}$ de $\Delta^{\prime \prime}(\star) \cup \Delta^{\prime \prime \prime}(\star)$ dans $\Delta_{\text {apol }} \subset \Delta_{p-1} \backslash \Delta^{\prime}$. Soit $\tilde{\Delta}=\Delta^{\prime \prime \prime}(\star) \cup\left(\Delta^{\prime}(\star) \cap \Delta^{\prime \prime}(\star)\right)$. On construit un difféomorphisme naturel de $\Delta_{p-1} \backslash\left(\Delta^{\prime} \cup \Delta^{\prime \prime}\right)$ sur $\Delta(r) \backslash\left(H^{\prime} \cup H^{\prime \prime}\right)$ induisant l'identité au voisinage de $\tilde{\Delta}$ comme suit. D'un côté on construit de manière analogue au cas précédent, un difféomorphisme de $\Delta_{p-1} \backslash \Delta^{\prime \prime}$ sur $\Delta_{\text {pol }} \backslash H^{\prime}$ induisant l'identité sur $U^{\prime}$ et de l'autre côté on construit de même un difféomorphisme de $\Delta_{p-1} \backslash \Delta^{\prime}$ sur $\Delta_{\text {apol }} \backslash H^{\prime \prime}$ induisant l'identité sur $U^{\prime \prime}$. Ces deux difféomorphismes 


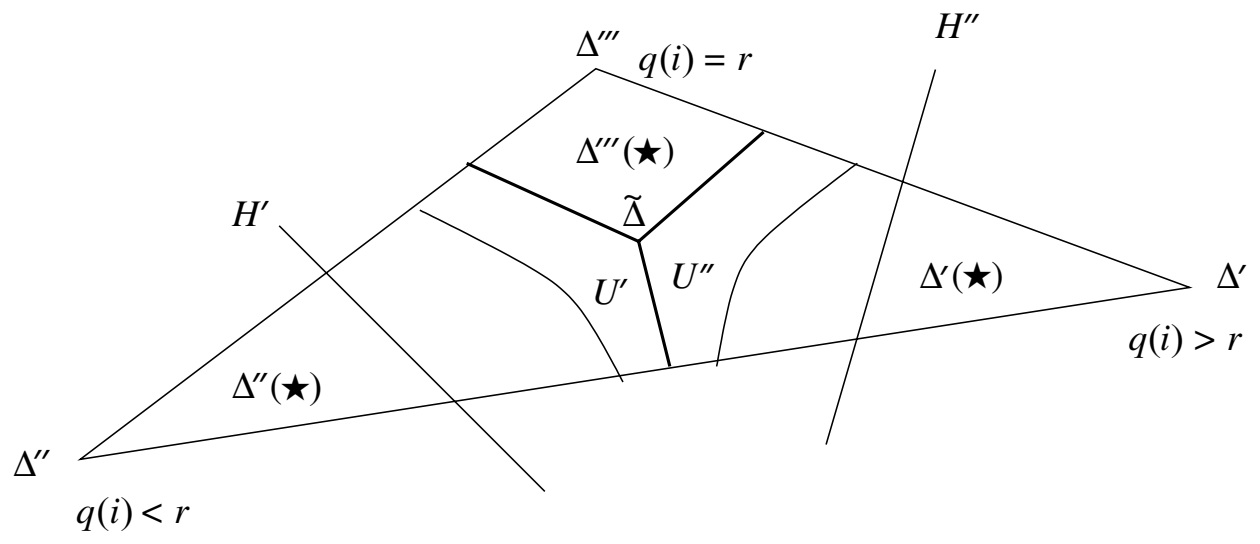

FiguRE 2.

induisent l'identité au voisinage $U^{\prime} \cap U^{\prime \prime}$ de $\tilde{\Delta}$, ce qui garantit que ce difféomorphisme soit compatible avec les recollements et se prolonge bien.

Quant à la rétraction elle se construit par les mêmes arguments. Revenons d'abord sur le difféomorphisme. Pour tout point $A \in \Delta^{\prime \prime}$, on considère le cône $C_{A}\left(\Delta^{\prime}, \Delta^{\prime \prime \prime}\right)$ des droites affines qui joignent le point $A$ à un point du joint des simplexes $\Delta^{\prime}$ et $\Delta^{\prime \prime \prime}$. Soit $D \in C_{A}\left(\Delta^{\prime}, \Delta^{\prime \prime \prime}\right)$ une telle droite. On choisit sur la droite $D$ un champ de vecteurs $\xi_{D}$ qui s'annule sur $D \cap U^{\prime}$ et dont le flot au temps 1 envoie le point $A$ sur le point $D \cap H^{\prime}$. La rétraction est alors construite de manière à envoyer le point $D \cap H^{\prime}$ sur le point d'intersection de la droite $D$ avec le squelette $\left(\Delta^{\prime}(\star) \cap \Delta^{\prime \prime}(\star)\right) \cup\left(\Delta^{\prime \prime}(\star) \cap \Delta^{\prime \prime \prime}(\star)\right) \cup\left(\Delta^{\prime}(\star) \cap \Delta^{\prime \prime \prime}(\star)\right)$. On procède de la même manière à partir d'un point $A \in \Delta^{\prime}$ en considérant le cône $C_{A}\left(\Delta^{\prime \prime}, \Delta^{\prime \prime \prime}\right)$. On obtient ainsi une rétraction de la zone $\Delta(r)$ correspondant à la couronne polaire sur $\tilde{\Delta}$ qui se relève en la rétraction recherchée, ce qui termine la démonstration de la proposition 1 et du théorème 1.

Notons le résultat suivant.

Proposition 2. Si le lieu polaire $C(\varphi \circ P)$ sur la désingularisée $Z$ passe par un point multiple $z \in D_{i_{1}} \cap \cdots \cap D_{i_{r}}$, les quotients d'Hironaka $q\left(i_{1}\right)=\cdots=q\left(i_{r}\right)$ sont nécessairement égaux.

Démonstration. Supposons qu'il existe deux indices $i_{1}$ et $i_{2}$ tels que $q\left(i_{1}\right) \neq q\left(i_{2}\right)$. Alors il suffit de démontrer que $C(\varphi \circ P) \cap D_{i_{1}} \cap D_{i_{2}}=\emptyset$.

D'après le lemme 2 , il existe des coordonnées analytiques complexes locales $x_{i}, i=1, \ldots, e$ telles que $f=x_{1}^{n_{1}} \cdots x_{e}^{n_{e}}$ et $g=x_{1}^{m_{1}} \cdots x_{e}^{m_{e}}$ où $e=\operatorname{dim} X$. Alors

$$
\partial f / \partial x_{i}=n_{i} x_{i}^{n_{i}-1} \prod_{j \neq i} x_{j}^{n_{j}} \quad \text { et } \quad \partial g / \partial x_{i}=m_{i} x_{i}^{m_{i}-1} \prod_{j \neq i} x_{j}^{m_{j}} .
$$

Dire que le rang de la matrice jacobienne $(\partial f / \partial x, \partial g / \partial x)$ est $\leqslant 1$ revient à annuler les mineurs de rang 2 , soit

$$
\left(n_{i_{1}} m_{i_{2}}-n_{i_{2}} m_{i_{1}}\right) \prod_{j \neq i_{1}, i_{2}} x_{j}^{n_{j}+m_{j}} x_{i_{1}}^{n_{i_{1}}+m_{i_{1}}-1} x_{i_{2}}^{n_{i_{2}}+m_{i_{2}}-1}=0
$$

en dehors de $D$; d'où $n_{i_{1}} m_{i_{2}}=n_{i_{2}} m_{i_{1}}$ et $q\left(i_{1}\right)=q\left(i_{2}\right)$, donc les quotients d'Hironaka sont tous égaux, ce qui est absurde.

Remarque. La filtration valuative se prête bien à l'étude par une projection sur le DCN, alors que la filtration polaire se prête à l'étude par la projection $\varphi$ dans $\mathbb{C}^{2}$, comme nous allons le voir. 


\section{B. Audoubert, F. El Zein et Lê Dũng Tráng}

\section{Théorie de Lefschetz sur la fibre de Milnor $F$ de $f$}

Considérons de nouveau $\varphi:(X, 0) \longrightarrow\left(\mathbb{C}^{2}, 0\right)$ satisfaisant les hypothèses faites au début. Nous allons généraliser ici un raisonnement se trouvant dans [Mau95] (respectivement [Tah97]) dans le cas où $X=\mathbb{C}^{2}$ (respectivement $X$ est une surface normale), qui montre comment l'on retrouve les nombres polaires sur la désingularisée de $X$. Dans leur cas le raisonnement repose sur la formule de Riemann-Hurwitz. En dimension supérieure, on remplace l'utilisation de la formule de RiemannHurwitz par la théorie de Lefschetz. Les points de $\Delta(\varphi) \cap(u=t)$ sont les valeurs critiques pour la restriction de $g$ à la fibre de Milnor $F_{t}$. D'après l'hypothèse faite (voir $\S 2.3$ ), la restriction de $g$ au-dessus du disque $D_{r}=D\left(0, B|t|^{r}\right)$ moins ces points est une fibration localement triviale.

Un calcul simple des caractéristiques d'Euler-Poincaré utilisant des techniques de la théorie de Lefschetz donne le résultat suivant :

Proposition 3. i) Soient $r$ un nombre polaire et $v_{\lambda}, \lambda \in \Lambda_{r}$, les valeurs critiques de la restriction de $g$ à la fibre de Milnor $F_{t}=f^{-1}(t)$ dans la couronne $C(r)$ (i.e. $A|t|^{r}<\left|v_{\lambda}\right|<B|t|^{r}$ ), w un point général de $\mathbb{C}^{2} \backslash(\Delta(\varphi) \cup(u v=0)), G_{v_{\lambda}}$ les fibres singulières $F_{t} \cap g^{-1}\left(v_{\lambda}\right)$ et $G_{w}$ la fibre générale. On a la formule suivante pour les caractéristiques d'Euler-Poincaré de la filtration polaire :

$$
\chi\left(g^{-1}(C(r)) \cap F_{t}\right)=\sum_{\lambda \in \Lambda_{r}} \chi\left(G_{v_{\lambda}}\right)-\chi\left(G_{w}\right) .
$$

ii) Si de plus, les points critiques au-dessus des $v_{\lambda}$ sont des points $x_{i}, i \in I_{\lambda}$, en nombre fini, le second terme dans (i) est donné par un calcul de Lefschetz en terme de la fibre de Milnor $F_{i}$ de la singularité $\left(G_{v_{\lambda}}, x_{i}\right)$ et de la variété évanescente $E v\left(x_{i}\right)$ :

$$
\chi\left(G_{v_{\lambda}}\right)-\chi\left(G_{w}\right)=\sum_{g\left(x_{i}\right)=v_{\lambda}} \operatorname{dim} E v\left(x_{i}\right)=\sum_{g\left(x_{i}\right)=v_{\lambda}}\left(1-\chi\left(F_{i}\right)\right) .
$$

\section{Application}

Le difféomorphisme stratifié entre les deux filtrations donne la relation souhaitée entre les invariants associés aux deux projections. Nous nous contenterons d'illustrer cette relation au niveau de la caractéristique d'Euler-Poincaré où les simplifications techniques de calcul permettent d'obtenir des formules relativement simples à énoncer.

Proposition 4. Supposons la condition de l'assertion ii de la proposition 3 du $\S 5$ vérifiée et soit $r$ un nombre polaire fixé. Alors en notant $n$ la dimension de $X$ et $\mu_{i}$ le nombre de Milnor d'une singularité isolée, on a l'égalité des caractéristiques d'Euler-Poincaré suivantes :

$$
\left.\chi\left(\Pi^{-1} \underline{(D(r)}\right)\right)=\chi\left(g^{-1}(C(r)) \cap F_{t}\right)=(-1)^{n-1} \sum_{\lambda \in \Lambda_{r}} \sum_{g\left(x_{i}\right)=v_{\lambda}} \mu_{i}
$$

où $v_{\lambda}$, avec $\lambda \in \Lambda_{r}$, parcourt les valeurs critiques de $\varphi$ dans la couronne $C(r)$ et

$$
\underline{D(r)}=\left(\bigcup_{q(i)=r} D_{i}\right) \cup\left(\bigcup_{q(k)>r, q(j)<r} D_{k} \cap D_{j}\right) .
$$

Démonstration. Soit $r$ un nombre polaire fixé. Si l'on note $\mu_{i}$ le nombre de Milnor [Mil68] de la singularité $\left(G_{v_{\lambda}}, x_{i}\right)$, on en déduit (voir $\S 5$ ) l'expression suivante pour la caractéristique d'EulerPoincaré de la couronne polaire, en notant $n$ la dimension de $X$,

$$
\chi\left(g^{-1}(C(r)) \cap F_{t}\right)=(-1)^{n-1} \sum_{\lambda \in \Lambda_{r}} \sum_{g\left(x_{i}\right)=v_{\lambda}} \mu_{i} .
$$




\section{INVARIANTS D'UNE DÉSINGULARISATION ET SINGULARITÉS DES MORPHISMES}

D'autre part d'après notre résultat principal (voir $\S 2.5$ ) la couronne polaire $g^{-1}(C(r)) \cap F_{t}$ et $\Pi^{-1}(D(r))$ ont le même type d'homotopie donc en particulier la même caractéristique d'EulerPoincaré.

Corollaire 1. Pour tout nombre polaire $r$ il existe un quotient d'Hironaka $q(i)$ avec $i \in I$ tel que $q(i)=r$.

Démonstration. La projection $\Pi$ étant une fibration stratifiée par des fibrés en tores au-dessus des points de multiplicité $>1$ la caractéristique d'Euler-Poincaré de $\Pi^{-1}\left(\bigcup_{q(k)>r, q(j)<r} D_{k} \cap D_{j}\right)$ est par conséquent nulle. Soit $r$ un nombre polaire fixé. Si l'on suppose que tous les quotients d'Hironaka sont distincts de $r$, alors $\chi\left(\Pi^{-1}(D(r))\right)=0$, ce qui est contradictoire avec la non nullité de la caractéristique d'Euler-Poincaré de la couronne polaire $g^{-1}(C(r)) \cap F_{t}$ donnée par la proposition précédente.

Corollaire 2. Pour toute composante irréductible $D_{i}$ soit

$$
D_{i}^{\star}=D_{i} \backslash \bigcup_{j \neq i} D_{j} .
$$

Pour tout nombre polaire $r$ on a

$$
\sum_{q(i)=r} v_{D_{i}}(f) \chi\left(D_{i}^{\star}\right)=(-1)^{n-1} \sum_{\lambda \in \Lambda_{r}} \sum_{g\left(x_{i}\right)=v_{\lambda}} \mu_{i} .
$$

Démonstration. La projection $\Pi$ induit au-dessus des points de multiplicité $s$, pour $s \geqslant 2$, un fibré dont les fibres sont homotopes à une réunion disjointe de tores $T^{s-1}$ et donc de caractéristique d'Euler-Poincaré nulle. Au-dessus d'une composante $D_{i}$ la projection $\Pi$ induit un revêtement fini de degré $v_{D_{i}}(f)$ sur $D_{i}^{\star}=D_{i} \backslash \bigcup_{j \neq i} D_{j}$. On en déduit que

$$
\chi\left(\Pi^{-1}(\underline{D(r)})\right)=\sum_{q(i)=r} v_{D_{i}}(f) \chi\left(D_{i}^{\star}\right) .
$$

Remarque. La condition de l'assertion ii de la proposition 3 est en général vérifiée quand par exemple le lieu critique $C(\varphi)$ de $\varphi$ est une courbe, ce qui est le cas pour $g$ une projection générique (voir $[\operatorname{Le} 77])$.

\section{BIBLIOGRAPHIE}

BK86 E. Brieskorn et H. Knörrer, Plane algebraic curves (Birkhäuser, Basel, 1986).

Cle69 C. H. Clemens, Picard-Lefschetz theorem for families of nonsingular algebraic varieties acquiring ordinary singularities, Trans. Amer. Math. Soc. 136 (1969), 93-108.

Cle77 C. H. Clemens, Degeneration of Kähler manifolds, Duke Math. J. 44 (1977), 215-290.

Le75 Lê D. T., La monodromie n'a pas de point fixe, J. Math. Tokyo Univ., Sec. 1A, 22 (1975), 409-427.

Le77 Lê D. T., Some remarks on relative monodromy, in Real and complex singularities (Proc. Ninth Nordic Summer School/NAVF Sympos. Math., Oslo, 1976), (Sijthoof and Noordhoof, Alphen aan den Rijn, 1977), 397-403.

Le78 Lê D. T., The geometry of the monodromy theorem, in C. P. Ramanujam - A tribute, Tata Institute of Fundamental Research Studies in Mathematics, vol. 8 (Springer, Berlin-New York, 1978), $157-173$.

LMW89 Lê D. T., F. Michel et C. Weber, Sur le comportement des courbes polaires associées aux germes de courbes planes, Compositio Math. 72 (1989), 87-113.

LT83 Lê D. T. et B. Teissier, Cycles évanescents, sections planes et conditions de Whitney II, in Proc. Symp. Pure Mathematics, vol. 40, part 2 (American Mathematical Society, Providence, RI, 1983), $65-103$. 


\section{INVARIANTS D'UNE DÉSINGULARISATION ET SINGULARITÉS DES MORPHISMES}

Mau95 H. Maugendre, Topologie des germes jacobiens, Thèse de doctorat, Université de Nantes (1995).

Mil68 J. Milnor, Singular points of complex hypersurfaces, Ann. Math. Stud., vol. 61 (Princeton University Press, 1968).

Mum61 D. Mumford, The topology of normal singularities of an algebraic surface and a criterion for simplicity, Publ. Math. Inst. Hautes Études Sci. 9 (1961).

SZ85 J. Steenbrink et S. Zucker, Polar curves, resolution of singularities and the filtered mixed Hodge structure on the vanishing cohomology, Lecture Notes in Mathematics, vol. 1273 (Springer, Berlin, 1985), 178-202.

Tah97 S. Taher, Sur le lieu polaire de morphismes analytiques complexes, C. R. Acad. Sci. Paris, Sér. I, 324 (1997), 439-442.

Tei75 B. Teissier, Introduction to equisingularity problems, in Proc. Symp. Pure Mathematics (Arcata 1974), vol. 29 (American Mathematical Society, Providence, RI, 1975), 593-632.

Tho70 R. Thom, Structure locale des morphismes analytiques, Actes du Congrès International des Mathématiciens, tome 2 (Gauthier-Villars, Paris, 1970), 257-265.

Benoît Audoubert baudou@math.metro-u.ac.jp

Department of Mathematics, University of Nijmegen, Toernooiveld 1, 6525 ED Nijmegen, The Netherlands

Current address: Department of Mathematics, Tokyo Metropolitan University, Minami-Ohsawa 1-1, Hachioji-shi, Tokyo 192-0397, Japan

Fouad El Zein elzein@math.univ-nantes.fr

Département de Mathématiques, Université de Nantes, 2, rue de la Houssinière, 44322 Nantes cedex 03, France

Lê Dũng Tráng ledt@gyptis.univ-mrs.fr

Centre de Mathématiques, Université de Provence, 39, rue F. Joliot Curie, 13453 Marseille cedex 13, France 